\title{
The histological effects of zinc oxide nanoparticles on the kidney of adult male rabbits
}

\author{
Hekmet O Abdel-Aziz,Eman E Ragab,,Hagar M Hamdan
}

\author{
Ass.Prof.of Histology, Facultyof Medicine, Sohag University \\ Lectuerof Histology, Facultyof Medicine, Sohag University \\ MD.of Histology, Facultyof Medicine, Sohag University
}

\begin{abstract}
:
Introduction:The growth of Nanotechnology in the past few years changes every aspects of human life. Today, nanoparticles are used in personal care products, cosmetics, sunscreens, and in biomedicine as drug delivery system and for therapeutic purposes.Among the most common nanotechnology in consumer and medical products are zinc oxide nanoparticles ( $\mathrm{ZnO}$ NPs). Most applications of ZnO NPs exploit the reactivity of the oxide, as a precursor to other zinc compounds. They are widely used as drug delivery vehicles, anticancer agents, components of the restorative dental and food packaging materials, and as cosmetic, antiseptic, and ultraviolet protection products (sun screen). Exposure to ZnO NPs could occur in the industrial settings and through everyday consumer products.
\end{abstract}

Aim of the work: Our aim was to study the histological changes in the kidney of adult male rabbit after administration of different doses of ZnO NPs.

Materials and method:Our study was carried out on 15 adult male rabbits. The animals were divided into three groups randomly. Group I: kept without treatment as control group.Group $\Pi$ : were received $\mathrm{ZnO} \mathrm{NPs}$ by intraperitonial injections in a dose of $100 \mathrm{ml} / \mathrm{kg}(0.4 \mathrm{~cm}$ of prepared solution $)$ once daily for 14 days.Group $\Pi \mathrm{I}$ : were received ZnONPs byintraperitonial injections in a dose of $250 \mathrm{ml} / \mathrm{kg}(1 \mathrm{~cm}$ of prepared solution) once daily for 14 days.The rabbits were sacrificed on the fifteenth day of the experiment,kidneys of each animal were obtained and stainrd by Hx\&E.

RESULTS:ZnO NPs have had a prominent toxic effects on kidney tissue. There were destruction of proximal convoluted tubules inform of loss of brush border, vacuolation of cytoplasm and intratubal protein deposition. Also the renal corpuscles showed congestion and dilation of glomerulus.

Conclusion: ZnO NPs causes histopathological changes of the kidney through oxidative stress. These may give us more information on hazards of $\mathrm{ZnO}$ NPs on human health.

\section{Introduction}

Nano-revolution impacts on every aspect of our life, medical, science, drug applications, and defense industries (Long and Ye, 2007).Nanoparticals (NPs) exposure is rapidly increasing led to a growing concern on the bioavailability and toxicity of NPs (Igarashi, 2008).
$\mathrm{ZnO}$ NPs are one of the most toxic metallic NPs.This due to its higher ability to shed ions and its high solubility(Watson et al., 2014).ZnO NPs produced marked cytotoxicity in several types of cell lines, as well as an apparant activation of signal transduction 
SOHAG MEDICAL JOURNAL The histological effects of zinc oxide nanoparticles on the kidney Vol. 22 No. 2 July 2018

Hagar M Hamdan

pathways induce apoptosis (Sharma et al., 2012,a).

Toxicity includes cytotoxic, genotoxic, and proinflammatory effects. ZnO NPs induce oxidative damage through reactive oxygen species (ROS) so it triggered mitochondrial mediate apoptosis. High dose of ZnO NPs could cause nephrotoxicity and alternation in kidney metabolism in experimental animal (Sharma et al., 2012,b).

Mitochondria are considered target organelles for cytotoxic injury. They have a critical role in maintaining cellular functions favoring aerobic ATP production. In addition to supplying cellular energy. The mitochondrion is one of the major cell targets for $\mathrm{ZnO}$ NPs-induced ROS and the intrinsic mitochondrial apoptotic pathway has an important role in $\mathrm{ZnO}$ NPs-induced cell death. Once $\mathrm{ZnO}$ NPs reach the mitochondria, they can generate mitochondrial ROS via impaired electron transport chain and depolarize mitochondrial membrane can catalyze the generation of superoxide $(\mathrm{O} 2)$ either by accelerating electron transfer to molecular oxygen or blocking the electron transport chain of mitochondria (Xiao et al., 2016).

\section{Materials and methods}

Animals:

The present study was set on 15 adult male rabbits (obtained from the animal house of Sohag University), weighing from 1.5 to $2 \mathrm{~kg}$. They were housed in clean properly ventilated cages under the same environmental conditions with free access to food and water throughout the whole period of experiment.

\section{Chemicals:}

\section{Results:}

Hematoxylin and eosin stain of control group: showed that the kidney was modified into cortex and medulla. The cortex is the outer zone and composed of renal corpuscles
Zinc oxide nanoparticle (>100nm) powder was obtained from SigmaAldrich chemicals. 60 gram of the powder was dissolved in $220 \mathrm{cc}$ of normal saline and dispersed by ultrasonic vibration for $15 \mathrm{~min}$.

\section{Experimental design:}

The Institutional Animal Care and the Research Ethics Committee of the Faculty of Medicine, Sohag University, Egypt, approved the experimental protocol.

The animals were divided into three groups randomly, 5 animals in each:

Group I: kept without treatment as control group.

Group $\Pi$ : were received ZnONPs byintraperitonial injections in a dose of $100 \mathrm{ml} / \mathrm{kg}(0.4 \mathrm{~cm}$ of prepared solution $)$ once daily for 14 days.

Group ПI: were received ZnONPs byintraperitonial injections in a dose of $250 \mathrm{ml} / \mathrm{kg}(1 \mathrm{~cm}$ of prepared solution) once daily for 14 days.

\section{Methods:}

The rabbits were sacrificed on the fifteenth day of the experiment after being anaesthetized by ether. Kidneys and both femur of each animal were obtained.

Kidneys were fragmented from $0,5-1,0$ $\mathrm{cm}$ in thickness, renised in saline and fixed in $10 \%$ formalin for 24 hours. The preserved organs were processed, dehydrated with alcohol, cleared with xylol, infiltrated and embedded with paraffin wax. Paraffin wax blocks were sectioned at $5 \mu$ thick with a rotary microtome using disposable blade. Sectioned slides were stained for Light microscopy:

A- Haematoxylin and Eosin for general structure 
SOHAG MEDICAL JOURNAL The histological effects of zinc oxide nanoparticles on the kidney Vol. 22 No. 2 July 2018 Hagar M Hamdan

(Bowman's capsule and glomerulus) and renal tubules proximal convoluted tubule (PT) and distal convoluted tubule (DT). PT was identified by its narrow lumen, number of cell in section about 3 to 4 cells and the apical part of the cell has brush border. Both PT \&DT were lined by simple cuboidal epithelium and had basal striation. Renal medulla was composed of collecting tubule and loop of Henles (Fig.1).

\section{Low dose treated group}

Sections of (group II) revealed histological changes as compared to the control group. The renal corpuscles were more or less similar to the normal with no changes. While there were disturbance in renal tubules. There were loss of their brush bored in large number of PT (Fig.2). There was vacuolation of the cytoplasm of some tubules; there was destruction of some of DT with dense nucleus(Fig.3).

\section{High dose treated group}

revealed more histological changes than the previous group. There were increase in the bowman's space with intact partial layer and congestion of intraglomerular capillaries (Fig.15). Proximal tubules lost their brush border and there was vacuolation of their cytoplasm (Fig.13\&14). There was destruction of some of DT (Fig.13). Also there were infiltrations of inflammatory cells and capillaries congestion in between tubules (Fig.16). There was intra tubular protein deposition (Fig.13).

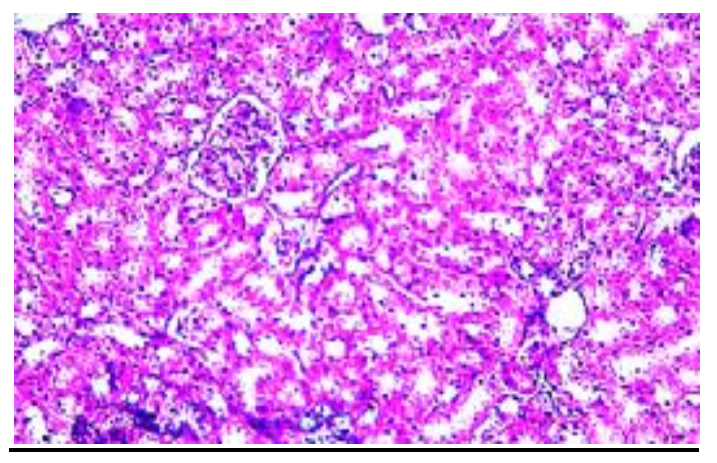

Fig(1): A photomicrograph of the control kidney showing, the normal architectuer of the kidney.the renal corpusles composed of glomerulus $(\mathrm{G})$ and surrounded by bowmanscapsule.the renal tubules modified into proximal (P ) and distal (D) tubule(H\&E 200).

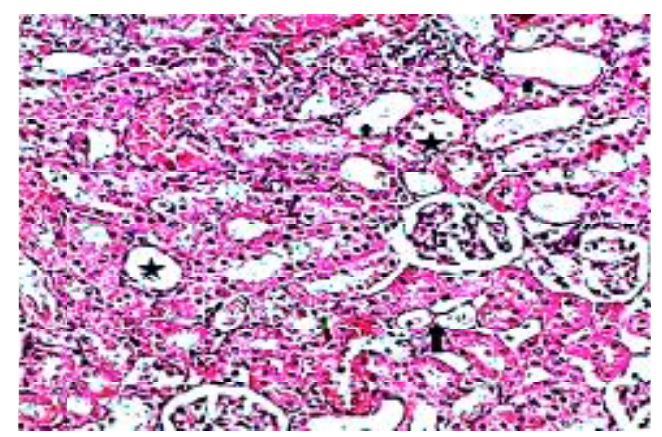

Fig(2): A photomicrograph of the kidney treated by $(100 \mathrm{mg} / \mathrm{Kg}$ of $\mathrm{ZnO} \mathrm{NPs})$ showing ,some of PT loss their brush border(stars), However some of them still have brush border (arrow) and some of DCT their nucleus appear dense (arrow heads) (H\& E 200). 


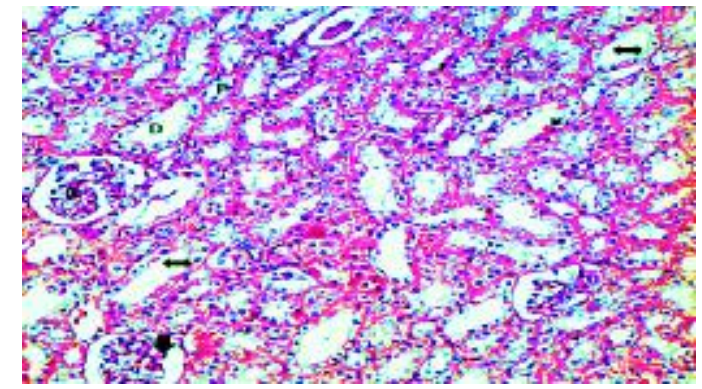

Fig. (3): A Photomicrograph of the kidney (treated by $250 \mathrm{mg} / \mathrm{Kg}$ of $\mathrm{ZnO} \mathrm{NPs}$ ) showing PCT loss their brush border and there is vacuolation of their cytoplasm (left-right arrows), and increase the bowman's space with intact parietal layer with congestion of intraglomerular capillaries (arrow head) (H \& E 200).

\section{Discussion}

NPs show high rate of bio distribution than ordinary particles .Single dose of $\mathrm{ZnO} \quad \mathrm{NPs} \quad(20-100 \mathrm{~nm})$ when intraperitoneally administrated at dose $(2.5 \mathrm{~g} / \mathrm{kg})$ resulted in accumulation of $\mathrm{ZnO} \mathrm{NPs}$ in different organs. Concentrations of $\mathrm{ZnO}$ NPs in the organs were higher after administration of similar amounts of $\mathrm{ZnO}$ particles $(\mathbf{L i}$ et al., 2012).

$\mathrm{ZnO}$ NPs have two major routes of elimination from the circulatory system through bile in the faeces or through the kidneys in the urine. Cho et al. (2013) who indicated that the zinc concentration in the urine of $\mathrm{ZnO}$ NPs treaedt groups significantly increased in a dose dependent manner. The exposure route influenced the rate of $\mathrm{ZnO}$ NPs clearance as $\mathrm{ZnO}$ NPs injected into the peritoneum persisted in the serum for a longer period of time than those administered orally (Li et al., 2012). $\mathrm{ZnO}$ NPs are one of the most toxic metallic NPs.This due to its higher ability to shed ions and its high solubility(Watson et al., 2014).ZnO NPs produced marked cytotoxicity in several types of cell lines, as well as an apparant activation of signal transduction pathways induce apoptosis. On the other hand, exposure to $\mathrm{ZnO}$ NPs shows a genotoxic potential mediated by lipid peroxidation and oxidative stress (Sharma et al., 2012,a).

The most prominent result in our study was loss of brush border of PCT. That was obviously appeared in our sections. These agree with lin et al. (2016) who founded histopathological changes of the kidneys in form of tubular dilatation, loss of brush borders and flattening of tubular epithelium in mice treated by $\mathrm{ZnO}$ NPs. This explained by ZnO NPs induce cytotoxictyand genotoxicity in kidney epithelial cells, as ROS generation and apoptosis may be the intrinsic reasons for the high toxicity (Uzar et al., 2015).

We observed also other signs of degenerations of the epithelial cells of renal tubules in the form of cytoplasmic vacuolation. This agrees with Tang et al. (2016) who found cytoplasmic vacuolation and swelling of the liver cell and renal tubules epithelial cells in the rat treated with $600 \mathrm{mg} / \mathrm{kg}$ of Zno NPs.

In our study, there were prominent intra tubular homogenous acidophilic depositions that appeared insections. This agrees with Ben-Slama et al. (2015) who describe these homogenous accumulations as intra tubular protein deposition. But other study they consider 
SOHAG MEDICAL JOURNAL The histological effects of zinc oxide nanoparticles on the kidney Vol. 22 No. 2 July 2018

these homogenous accumulations as hemorrhage inside renal tubules.

In addition, there was apparent blood congestion in between tubules and intraglomerular capillaries. This result was supported by Ben-Slama et al., (2015) who noticed appearance of sinusoidal congestion, RBC deposition in the liver veins.

$\mathrm{ZnO}$ NPs had a severe renal toxicological effect and led to increase of blood biochemical markers (blood urea nitrogen and creatinine) suggested the renal dysfunction (Najafzadeh et al., 2013). Thus we concluded that high dose of $\mathrm{ZnO}$ NPs lead to its accumulation in kidney and cause renal structureal damage.

Conclusion: $\mathrm{ZnO}$ NPs have had cytotoxic effect on the kidney in dose dependent manner.

\section{References:}

1-Ben-Slama I, Mrad I, Rihane N, Mir LE, Sakly M, et al (2015): Sub-Acute Oral Toxicity of Zinc Oxide Nanoparticles in Male Rats. J NanomedNanotechnol 6: 284.

2-Cho WS, Duffin R, Bradley M, Megson IL, MacNee W, Lee JK, Jeong J, Donaldson K. (2012): Predictive value of in vitro assays depends on the mechanism of toxicity of metal oxide nanoparticles. Part FibreToxicol; 10-55.

3-Igarashi E. (2008): Factors affecting toxicity and efficacy of polymeric nanomedicines. Toxicol. Appl. Pharmacol. 229 (1), 121-134.

4-Long, X.F., Ye, Z.G. (2007):Relaxor behavior in $\mathrm{Ba}(\mathrm{Zn} 1 / 3 \mathrm{Nb} 2 / 3) \mathrm{O}-3-$ $\mathrm{PbTiO} 3$ new solid solution. IEEE Trans. Ultrason. Ferroelectr. Freq. Control 54 (12), 2595-2598.

5-Li C, Shen C, Cheng Y, Huang $\mathrm{H}$, Wu C, Kao C, Liao J, Kang J.(2012): Organ bio distribution, clearance, and genotoxicity of orally administered zinc oxide nanoparticles in mice. Nanotoxicology; 6:746-756.

6-Lin Y, Chiu I, Cheng F, Lee Y, Wang Y, Hsu Y et al.(2016): The role of hypoxiainducible factor-1alpha in zinc oxide nanoparticle-induced nephrotoxicity in vitro and in vivo. Part FibreToxicol :1352.

7-Najafzadeh H, Ghoreishi S, Mohammadian B, Rahimi E, Afzalzadeh MR, Kazemivarnamkhasti M, Ganjealidarani H. (2013):Serum biochemical and histopathological changes in liver and kidney in lambs after zinc oxide nanoparticles administration, Vet World 6(8): 534-537.

8-Sharma V, Anderson D, Dhawan A. (2012,a): Zinc oxide nanoparticles induce oxidative DNA damage and ROStriggered mitochondria mediated apoptosis in human liver cells (HepG2). Apoptosis; 17:852-870.

9-Sharma V,Singh P, Pandey A, Dhawan A. $(2012, b)$ : Induction of oxidative stress,DNA damage and apoptosis in mouse liver after jsub-acute oral exposure to zinc oxide nanoparticles. Mutat Res 745: 84-91.

10-Tang H, Min X, Qian R, Ru-Wen J, Qi-Ji L, Ying-Lun L. (2016): The effect of $\mathrm{ZnO}$ nanoparticles on liver function in rats .Int J Nanomedicine. : 4275-4285.

11-Uzar N, Abudayyak M, Akcay N, Algun G, Ozhan G. (2015): Zinc oxide nanoparticles induced cyto- and genotoxicity in kidney epithelial cells. ToxicolMech Methods; 25:334-9.

12-Watson C, Ge J, Cohen J, Pyrgiotakis G, Engelward BP, Demokritou $P$. (2014):.High-throughput screening platform for engineered nanoparticlemediated genotoxicity using Comet Chip technology. ACS Nano; 8:2118-2133.

13-Xiao L, Chunhua L, Xiaoniao C, Zhuo Y. (2016): Zinc oxide nanoparticles induce renal toxicity through reactive oxygen species, Food and Chemical Toxicology 90102echnology. ACS Nano; 8:2118-2133. 